\section{The value of lacrimal scintillography in the assessment of patients with epiphora}

OA Vonica ${ }^{1,2}$, E Obi $^{1,2}$, Z Sipkova $^{1,2}$, C Soare $^{3}$ and AR Pearson ${ }^{1,2}$
${ }^{1}$ Department of Ophthalmology, Royal Berkshire Hospital NHS Foundation Trust, Reading, UK

${ }^{2}$ Department of Oculoplastics, Prince Charles Eye Unit, Windsor, UK

${ }^{3}$ Department of Ophthalmology, King's College Hospital NHS Foundation Trust, London, UK

Correspondence:

OA Vonica, Department of Ophthalmology, Royal Berkshire Hospital NHS Foundation Trust, Reading RG1 5AN, UK

Tel: +44 (0)118322 5111; Fax: +44 (0)118322 8148. E-mail: oana.vonica@ nhs.net

Received: 1 September 2016 Accepted in revised form: 19 December 2016 Published online: 3 March 2017

\begin{abstract}
Purpose To assess the influence of dacryoscintillography (DSG) on the treatment decision for patients with epiphora and clinically patent non-functioning lacrimal systems.

Methods A retrospective 3-year review. Inclusion: patients having DSG for epiphora with delayed tear clearance, lacrimal system patency on syringing, and no visible external cause for watering. On the basis of regurgitation during syringing, tear ducts were divided into freely patent $(\mathrm{FP} \leq \mathbf{2 0} \%)$ or stenosed. The DSG results were examined for correlation with symptoms and clinical examination, the influence on decision to proceed to dacryocystorhinostomy (DCR), and the ability to predict the surgical outcome.

Results A total of 242 eyes were examined. The clinical diagnosis was FP in $45.5 \%$, nasolacrimal duct stenosis (NLDS) in $26.4 \%$, and other in $3.3 \%$. The DSG was normal in $30.9 \%$ of FP and $18.7 \%$ of NLDS eyes. Of the asymptomatic eyes, $46.7 \%$ had an abnormal DSG. DSG sensitivity was $73.6 \%$ and specificity $53.3 \%$. There was no significant difference in DSG results in those with FP or NLDS.

DCR was recommended in $39.1 \%$ of the symptomatic eyes with abnormal DSG. DCR surgery was considered inappropriate in all 46 eyes with normal DSG. DCR was successful in $76.5 \%$, however, the DSG result did not affect the success of surgery. Conclusion DSG has severe limitations due to lack of correlation with symptoms and clinical examination, inability to separate lacrimal duct narrowing from lacrimal pump function, and inability to predict the results of surgery. DSG can at best provide limited guidance on whether to proceed to DCR surgery.

Eye (2017) 31, 1020-1026; doi:10.1038/eye.2017.20; published online 3 March 2017
\end{abstract}

\section{Introduction}

Assessment and management of impaired tear drainage in patients whose lacrimal systems are patent remains challenging. In the absence of tear hypersecretion and once visible causes of pump failure have been excluded, there remains a substantial proportion of patients with epiphora who have impaired tear clearance yet patent tear passages on lacrimal syringing. Despite anatomical patency, the tear ducts in these patients appear to show physiological obstruction or stenosis. Although the results of dacryoctorhinostomy (DCR) surgery have been reported as lower than in those with complete obstruction, $50-94 \%$ of these patients will still benefit from surgery. ${ }^{1-7}$ Dacryoscintillography (DSG) examination has been used to guide the decision on whether surgery is appropriate, yet previous studies have considerable variation in the technique, normative data, analysis, and interpretation of this investigation, ${ }^{8}$ and the exact place of DSG therefore remains unknown. Our study aimed to help define the value of DSG by examining the correlation of the test with patient symptoms and clinical examination, its influence on management, and whether it predicts success of DCR surgery.

\section{Subjects and methods}

Our data were collected retrospectively over a 3-year period (2012-2014) from all patients attending specialist lacrimal clinics who had DSGs. Patients had a full lacrimal workup including tear meniscus height, fluorescein disappearance test, and tear duct syringing using a $2 \mathrm{ml}$ syringe and 26 gauge cannula by a single experienced surgeon (ARP). If there was significant regurgitation, the cannula was advanced to exclude canalicular obstruction or stenosis, and irrigation repeated from within the sac. Depending on the proportion of fluid reflux during syringing, the lacrimal systems were 
classified into two groups: freely patent (FP) if syringing was patent with regurgitation $\leq 20 \%$ or nasolacrimal duct stenosis (NLDS) if regurgitation on syringing was $>20 \%$. Patients with previous lacrimal surgery other than punctoplasty or evidence of canalicular disease were grouped separately. In unilateral cases, information on the asymptomatic fellow eye was also recorded. DSG examination was performed on all patients who had epiphora, clinical evidence of delayed tear clearance, no externally visible cause for watering (in particular, no ocular surface disease, punctual stenosis, or abnormalities of eyelid tone, position, or movement), and patent lacrimal systems on syringing. All the DSGs included in the study were performed pre DCR as part of the epiphora assessment. From all the DSGs performed during the study period, three were carried out post DCR and were not included in the analysis; these three post DCR DSGs were performed when epiphora symptoms persisted after O'Donoghue tube removal.

Our DSG technique has been described previously. ${ }^{9}$ Briefly, subjects were positioned upright with the nose in contact with the parallel hole collimator of a Mediso, Nucline X-ring gamma camera (Budapest, Hungary), and $4 \mathrm{MBq}( \pm 10 \%)$ technetium-99m per-technetate in $25 \mu \mathrm{l}$ isotonic saline was simultaneously instilled in the inferior fornix of both eyes. Dynamic imaging was started immediately and continued every minute for the first $10 \mathrm{~min}$, followed by static imaging at 15, 20, 25, 30, and $45 \mathrm{~min}$. All DSGs were reported by the same experienced observer regarding the time of entry of the tracer into the sac, duct, and nose, and subsequently reevaluated blinded to patient information to assess intra-observer consistency. The results were classified as showing normal or delayed transit according to the time for the tracer to reach the nose: up to $5 \mathrm{~min}$ normal, 6-15 $\mathrm{min}$ mild delay, 16-30 min moderate delay, 31-45 min severe delay, and $>45$ min very severe delay. ${ }^{10-12}$

\section{Statistical analysis}

Shapiro-Wilk, D'Agostino and Kolmogorov-Smirnov tests were performed to assess the distribution of continuous variables. Categorical variables are presented as proportions and continuous variables as means $\pm 1 \mathrm{SD}$ if they were normally distributed, and as median and interquartile range if they were not. Parametric or nonparametric statistical tests were used depending on the distribution. $\chi^{2}$-test was used to compare proportions for categorical variables.

Sensitivity, specificity, positive predictive value (PPV), and negative predictive value (NPV) were calculated using a two-by-two contingency table; confidence intervals (CIs) were calculated using Wilson-Brown method. Results were considered statistically significant if the one-sided $P$-value was $<0.05$.

\section{Results}

Over the 3-year period, DSG examination was performed on 130 patients. The files for nine patients were not available for analysis. The results of a total of 242 lacrimal systems in 121 patients were examined. The mean age was $60(\mathrm{SD}=15$ years), range 17-93 years (FP group mean age $59 \pm 16$, NLDS group $62 \pm 14$, and asymptomatic group $61 \pm 14$ ); $59.5 \%$ were females.

From the total of 242 eyes, the clinical diagnosis was FP in 110 eyes (45.5\%), NLDS in 64 eyes $(26.4 \%)$, and other in 8 eyes $(3.3 \%)-5$ canalicular disease and 3 post DCR. The patients with unilateral epiphora generated 60 asymptomatic eyes $(24.8 \%)$ for inclusion, with regurgitation $\leq 20 \%$ in $90 \%$.

\section{DSG correlation with the patient symptoms}

Despite epiphora, the DSG was normal in 34 out of $110 \mathrm{FP}$ eyes $(30.9 \%)$ and 12 out of 64 NLDS eyes (18.7\%); overall 46 out of $174(26.4 \%)$ of the symptomatic eyes had a normal test. Of the asymptomatic eyes, 28 out of 60 (46.7\%) had an abnormal DSG, with 11 out of 60 (18.3\%) having severe or very severe delay compared to 83 out of $174(47.7 \%)$ of symptomatic eyes.

The DSG test sensitivity was 73.6\% (95\% CI $=66.5-$ $79.6 \%)$ and the specificity $53.3 \%(95 \% \mathrm{CI}=40.9-65.4 \%)$.

DSG had a PPV of $82.0 \%(95 \% \mathrm{CI}=75.3-87.3 \%)$ and a NPV of $41.0 \%$ (95\% CI $=30.8-51.1 \%)$. The $2 \times 2$ contingency table used to calculate the sensitivity, specificity, PPV, and NPV is presented in Table 1. Reevaluation of the DSGs revealed no cases where the categorisation changed between assessments.

\section{DSG correlation with the clinical examination}

Compared to those with FP, there were fewer normal DSGs and greater levels of DSG delay in those with NLDS, however, the difference was not statistically significant (Table 2).

Comparing the actual tracer time to the nose, there was no significant difference between the NLDS and FP groups ( $P=0.21$, one-sided Kruskal-Wallis test). However, there was a significant difference between both

Table 1 Correlation between DSG and clinical examination

\begin{tabular}{lccc}
\hline DSG & Symptomatic & Asymptomatic & Total \\
\hline Abnormal DSG & 128 & 28 & 156 \\
Normal DSG & 46 & 32 & 78 \\
Total & 174 & 60 & 234 \\
\hline
\end{tabular}


Table 2 Correlation between DSG and clinical examination

\begin{tabular}{lccc}
\hline DSG & $\begin{array}{c}\text { FP (number of } \\
\text { eyes, \%) }\end{array}$ & $\begin{array}{c}\text { NLSD (number of } \\
\text { eyes, \%) }\end{array}$ & P-value \\
\hline Normal DSG & $34(30.9 \%)$ & $12(18.7 \%)$ & 0.079 \\
Mild delay & $18(16.4 \%)$ & $10(15.6 \%)$ & 0.890 \\
Moderate delay & $10(9.1 \%)$ & $7(10.9 \%)$ & 0.700 \\
Severe delay & $8(7.3 \%)$ & $6(9.4 \%)$ & 0.625 \\
Very severe & $40(36.4 \%)$ & $29(45.3 \%)$ & 0.249 \\
delay & & & \\
Total & 110 & 64 & \\
\hline
\end{tabular}

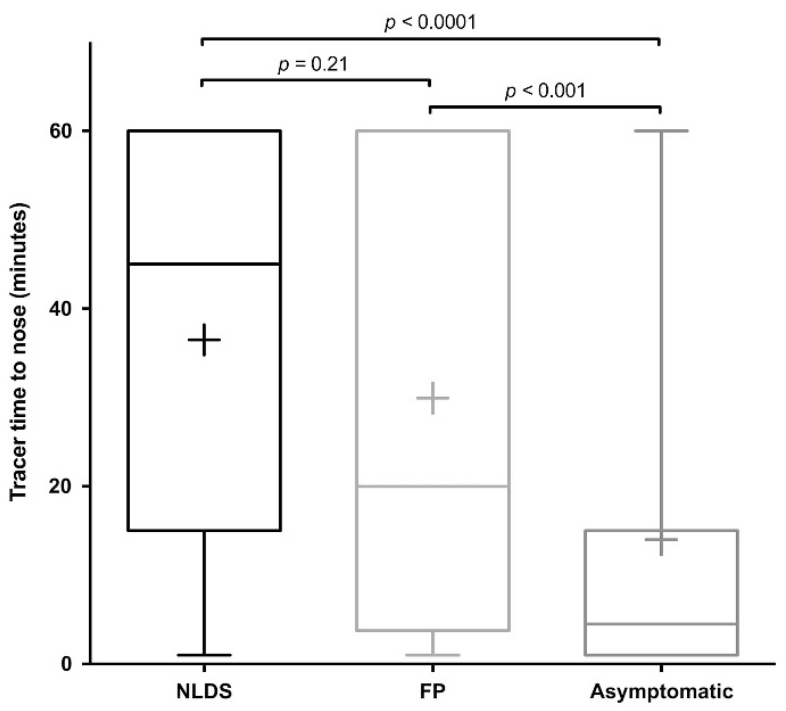

Figure 1 Tracer time to nose (minutes) showing mean (cross), median (line in box), 75th and 25th percentiles (top and bottom of the box), and maximum and minimum values.

these groups and the asymptomatic eyes $(P<0.0001$ and $P<0.001$, respectively, one-sided Kruskal-Wallis with multiple Dunn's comparison test) (Figure 1).

\section{Does the DSG result influence the management outcome?}

In our practice, DCR surgery was considered inappropriate in all the 46 out of $174(26.4 \%)$ symptomatic eyes with a normal DSG. Overall, DCR was

recommended in 50 out of 128 (39.1\%) of the symptomatic eyes with abnormal DSG (21 out of $76(27.6 \%)$ in the FP group and 29 out of 52 (55.8\%) in the NLDS group). Table 3 shows the proportions being recommended DCR surgery according to severity of delay in FP and NLDS groups. The most common reasons why DCR was not recommended despite symptoms and an abnormal DSG were: patients considered their symptoms manageable in 23 out of $128(17.9 \%)$, clinical picture remained inconsistent or unclear in 16 out of $128(12.5 \%)$, and when at the second clinical review, alternative treatment such as lid surgery or medical treatment was considered initially more appropriate in 14 out of 128 (10.9\%). Other reasons were: patient not keen for surgery, referred to ENT, lost at follow-up, and non-attendance.

Examples of DSGs and comparison with the clinical assessment demonstrating consistency and inconsistency are shown in Figure 2.

\section{Does the DSG result predict the success of DCR surgery?}

DCR success was established based on patient symptoms and clinical examination. Surgery was considered successful when there was no epiphora or minimal symptoms post operation with a patent tear duct, partially successful when there was improvement with some residual watering, and unsuccessful when symptoms were the same as before surgery or worse.

DCR surgery proceeded in 36 out of $50(72.0 \%)$ of the eyes for which it was indicated (15 out of 21 (71.4\%) in the FP group and 21 out of 29 (72.4\%) in the NLDS group). All DCR surgeries were performed endonasally with endoscope and bone punches. Reasons for nonproceeding to DCR included: patient did not attend in 2 out of $14(14.3 \%)$, medical reason in 5 out of $14(35.7 \%)$, patient cancelled or postponed in 4 out of $14(28.6 \%)$, and 3 out of $14(21.4 \%)$ were still awaiting surgery at the time of the study. In addition, the outcomes for 2 out of 36 eyes were unavailable due to loss to follow-up. Thus, a total of 34 eyes that had DCR surgery had a known outcome with results shown in Table 4. Mean follow-up was 18.2 \pm 17.36 weeks. The success rate in those with mild or moderate delay was $100 \%$ in both FP and NLDS groups. In those with severe or very severe delay, the success rate was $69 \%$ (FP 67\% and NLDS 71\%), with partial success in a further $24 \%$. The overall success rate was $76 \%$, with partial success in a further $21 \%$. There was no significant difference in success between the FP and NLDS groups $(P=0.81)$. Clinical examination revealed tear duct patency in all of the eight eyes with partial success or failure, but partial closure of the ostium in one for which further surgery was recommended. There was no significant difference in the mean tracer to nose time in the successful DCR eyes compared with the partially successful/failure DCR eyes $(P=0.18$, Mann-Whitney test).

\section{Discussion}

Since the technique was introduced in 1972 by Rossomondo et al, ${ }^{13}$ DSG examination has been used to help assess the cause of epiphora. There is recognition that in practice this test provides little extra information beyond clinical examination in those with complete lacrimal obstruction. ${ }^{10}$ However, its value in investigating those with patent but non-functioning lacrimal systems remains unclear, and there is very little information on the 
Table 3 Indication for DCR in FP and NLDS symptomatic eyes by severity of delay

\begin{tabular}{|c|c|c|c|c|c|}
\hline \multirow[t]{2}{*}{ DSG delay } & \multicolumn{2}{|r|}{$F P$} & \multicolumn{2}{|r|}{ NLDS } & \multirow[t]{2}{*}{ P-value } \\
\hline & Symptomatic eyes & $\begin{array}{l}\text { Eyes for which DCR was } \\
\text { recommended (number of eyes, \%) }\end{array}$ & Symptomatic eyes & $\begin{array}{l}\text { Eyes for which DCR was } \\
\text { recommended (number of eyes, \%) }\end{array}$ & \\
\hline Mild delay & 18 & $4(22.2 \%)$ & 10 & $3(30.0 \%)$ & 0.654 \\
\hline Moderate delay & 10 & $2(20.0 \%)$ & 7 & $3(42.8 \%)$ & 0.324 \\
\hline Severe delay & 8 & $0(0 \%)$ & 6 & $4(66.7 \%)$ & 0.008 \\
\hline $\begin{array}{l}\text { Very severe } \\
\text { delay }\end{array}$ & 40 & $15(37.5 \%)$ & 29 & $19(65.5 \%)$ & 0.023 \\
\hline Total & 76 & $21(27.6 \%)$ & 52 & $29(55.6 \%)$ & 0.001 \\
\hline
\end{tabular}

influence of the DSG result on a decision to proceed to DCR surgery nor whether the DSG findings can predict the results of surgery. Our results, to our knowledge, the largest reported study of this group of patients, show a poor correlation with symptoms. A significant proportion of symptomatic eyes have a normal result and a very high proportion of asymptomatic eyes have an abnormal result. The test specificity is low at $53.3 \%$ and the sensitivity only $73.6 \%$. Previous studies have shown abnormality rates up to $65 \%$ in fellow asymptomatic eyes of eyes with epiphora $9,14,15$ and a wide variation in transit times in normal eyes has also been seen. ${ }^{10-12}$ The high abnormal DSG rate in asymptomatic fellow eyes may partly reflect the commonly bilateral nature of tear duct disease, and the DSG may therefore be detecting subclinical narrowing of the tear passages in some of these eyes. There is some evidence that a proportion of these eyes later become symptomatic. ${ }^{16}$

The correlation of the DSG result with clinical examination was also poor with no significant difference in the proportion abnormal, the degree of abnormality, or the transit times of the FP and NLDS groups. Distinction between these groups inevitably relies on a subjective assessment of the proportion of fluid regurgitation during tear duct syringing. Nevertheless, the fact that this was carried out by an experienced surgeon, that the results were divided into just two broad groups (free and narrowed), and that in both these groups, the DSG transit times were significantly slower than in the asymptomatic eyes, indicates that the DSG results do not accurately reflect the degree of tear duct narrowing alone. Similarly, high abnormality rates have previously been noted by Peter $(77 \%$ with minimal resistance and $88 \%$ with $>20 \%$ regurgitation $)^{9}$ and Jabbour (66\% of those with minimal resistance), ${ }^{17}$ and Jager ${ }^{18}$ did not find a difference in quantitative DSG results according to syringing result, even when patients with complete obstruction were included.

The DSG result did influence the decision to recommend DCR surgery, in two ways. First, we felt it was difficult to justify proceeding to DCR where the DSG result is normal and therefore did not recommend surgery for the 46 eyes with normal DSG findings. Nevertheless, if accepted that there is a wide range of normal transit times, it is possible that a proportion of these patients may also benefit from surgery. Second, those with severe or very severe delay were twice as likely to have surgery recommended compared to those with mild or moderate delay. However, the clinical diagnosis had an equal influence with twice as many patients with an abnormal DSG having surgery recommended where the diagnosis was NLDS compared to FP, regardless of the severity of the DSG delay. We are unable to find any further studies that examine the practical influence of the DSG result on a decision to proceed to DCR surgery. Although there is some evidence that the location of hold-up can influence the outcome of DCR surgery, with higher success in those with post-sac delay compared to pre-sac delay, ${ }^{6}$ our results are the first to compare the results of surgery with the severity of the DSG abnormality. It is known that success rates of DCR tend to be higher in those with complete obstruction ${ }^{19-21}$ compared to those with non-functioning but patent systems, $3,4,7,22,23$ so higher levels of DSG delay would be expected to be associated with a higher surgical success rate. Although our overall success rate of $76.5 \%$ is comparable to previous studies, the severity of delay on DSG did not predict a successful outcome, again strongly suggesting that delayed transit is influenced by more than tear duct narrowing.

Several factors may explain the inconsistency of the DSG result with the patient's symptoms, the clinical assessment, and the outcome of surgery. As mentioned, there appears to be wide variation in transit times in normal, asymptomatic eyes. This may be due to natural physiological variation. However, an accurate result assumes normal tear production to provide sufficient flow of tears down the tear duct. It is known that transit times are higher in patients with dry eye $\mathrm{e}^{24}$ and it is therefore possible that a reduction in tear production, for example, with age, may influence the result. Similarly, increased tear production may mask lacrimal narrowing. It is also 


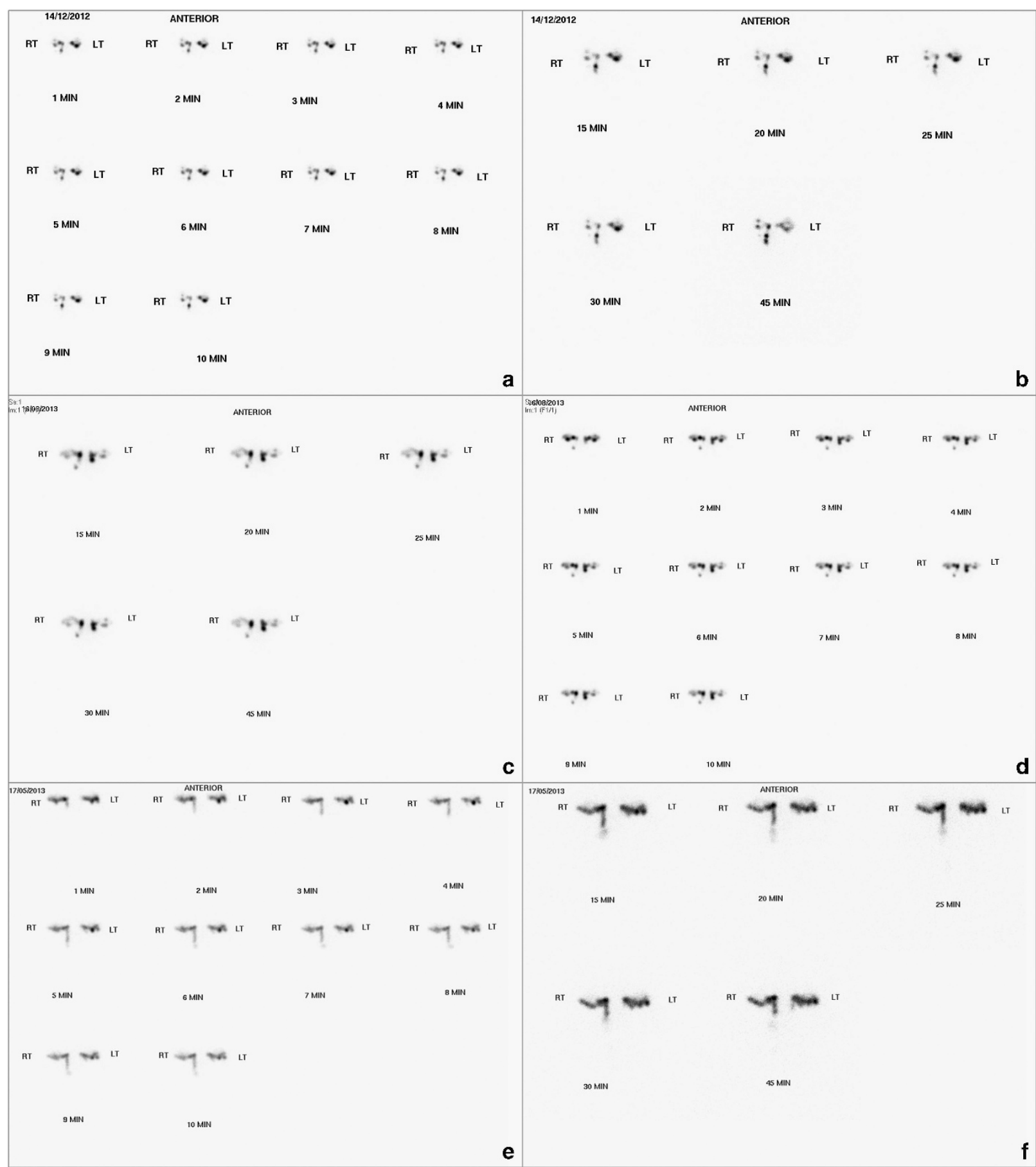

Figure 2 Top row—female 65 years old, only left eye symptomatic DSG showing consistency: left delay, right normal (a—early frames and $\mathrm{b}$-late frames). Middle row—male, 65 years old, only right eye symptomatic DSG showing lack of consistency: right normal, left delay (c-early frames and d-late frames). Bottom row-male, 65 years old, symmetrical epiphora, DSG showing lack of consistency with normal right, delayed left (e—early frames, $\mathrm{f}$-late frames).

known that tear fluid is absorbed in transit down the lacrimal duct ${ }^{22}$ and variation in the rate of absorption between patients may therefore also affect the rate at which fluid reaches the nasal space. Perhaps most importantly, the DSG assumes a normal lacrimal pump mechanism with the speed of drainage being influenced by a composite of lacrimal resistance and pump activity, among other possible factors. Although we excluded as far as possible factors that could affect the lacrimal pump, it remains possible that the abnormalities noted could in 
Table 4 Outcome of DCR surgery for 34 eyes

\begin{tabular}{|c|c|c|c|c|c|}
\hline \multirow[t]{2}{*}{ DSG delay } & \multicolumn{2}{|c|}{$\begin{array}{c}F P \\
\text { Total }=14\end{array}$} & \multicolumn{2}{|c|}{$\begin{array}{c}\text { NLDS } \\
\text { Total }=20\end{array}$} & \multirow[t]{2}{*}{ P-value } \\
\hline & $\begin{array}{c}\text { Success } \\
\text { (number of eyes, \%) }\end{array}$ & $\begin{array}{l}\text { Partial success/failure } \\
\text { (number of eyes, \%) }\end{array}$ & $\begin{array}{c}\text { Success } \\
\text { (number of eyes, \%) }\end{array}$ & $\begin{array}{l}\text { Partial success/failure } \\
\text { (number of eyes, \%) }\end{array}$ & \\
\hline Total & $11(78.6 \%)$ & $3(21.4 \%)$ & 15 (75.0\%) & $5(25.0 \%)$ & 0.81 \\
\hline Mild & $3(100 \%)$ & $0(0 \%)$ & $1(100 \%)$ & $0(0 \%)$ & \\
\hline Moderate & $2(100 \%)$ & $0(0 \%)$ & $2(100 \%)$ & $0(0 \%)$ & \\
\hline Severe & - & - & $1(50.0 \%)$ & $1(50.0 \%)$ & \\
\hline Very severe & $6(66.7 \%)$ & $3(33.3 \%)$ & $11(73.3 \%)$ & $4(26.7 \%)$ & \\
\hline
\end{tabular}

some cases be due to undetectable pump failure rather than tear duct narrowing. These are likely to persist or even worsen following surgery, ${ }^{23,24}$ and could account for the higher failure rate of DCR in patients with patent lacrimal systems compared to those with complete obstruction, even where surgery is anatomically successful. Faced with an abnormal DSG result, current techniques are unable to distinguish whether this reflects narrowing of the lacrimal passages, lacrimal pump dysfunction, or some other factor. Although some advocate dacryocystography as an alternative or complimentary investigation, it is a much less physiological test, is less sensitive, and correlates less closely with the clinical examination. ${ }^{9}$ Both tests still involve specialist equipment, are time consuming, and subject the patient to radiation.

In summary, our results show that, in patients with patent but non-functioning lacrimal systems, the lack of correlation with both symptoms and clinical examination, the inability to separate lacrimal duct narrowing from lacrimal pump function, and the inability to predict the results of surgery are severe limitations that mean DSG examination can at best provide only limited guidance on whether to proceed to DCR surgery.

\section{Summary}

What was known before

- DSG is widely used to investigate tear duct function, however, there is little consensus on its value in guiding a decision toward surgery.

\section{What this study adds}

- DSG examination has severe limitations due to lack of correlation with both symptoms and clinical examination, inability to separate lacrimal duct narrowing from lacrimal pump function, and inability to predict the results of surgery.

- DSG examination can at best provide only limited guidance on whether to proceed to DCR surgery.

\section{Conflict of interest}

The authors declare no conflict of interest.

\section{References}

1 Peter NM, Pearson AR. External dacryocystorhinostomy for the treatment of epiphora in patients with patent but nonfunctioning lacrimal systems. Br J Ophthalmol 2010; 94(2): 233-235.

2 O'Donnell BA, Clement CI. Assessing patients with epiphora who are patent to syringing: clinical predictors of response to dacryocystorhinostomy. Ophthal Plast Reconstr Surg 2007; 23(3): 173-178.

3 O'Donnell B, Shah R. Dacryocystorhinostomy for epiphora in the presence of a patent lacrimal system. Clin Exp Ophthalmol 2001; 29(1): 27-29.

4 Zaidy F, Symanski S, Olver JM. Endoscopic and External Dacrocystorhinostomy in the Management of Functional Epiphora. ESOPRS, Santiago de Compostela: Spain, 2001.

5 Moore WM, Bentley CR, Olver JM. Functional and anatomic results after two types of endoscopic endonasal dacryocystorhinostomy: surgical and holmium laser. Ophthalmology 2002; 109(8): 1575-1582.

6 Delaney YM, Khooshabeh R. External dacryocystorhinostomy for the treatment of acquired partial nasolacrimal obstruction in adults. Br J Ophthalmol 2002; 86(5): 533-535.

7 Sahlin S, Rose GE. Lacrimal drainage capacity and symptomatic improvement after dacryocystorhinostomy in adults presenting with patent lacrimal drainage systems. Orbit 2001; 20(3): 173-179.

8 Sagili S, Selva D, Malhotra R. Lacrimal scintigraphy: 'interpretation more art than science'. Orbit 2012; 31(2): 77-85.

9 Peter NM, Pearson AR. Comparison of dacryocystography and lacrimal scintigraphy in the investigation of epiphora in patients with patent but nonfunctioning lacrimal systems. Ophthal Plast Reconstr Surg 2009; 25(3): 201-205.

10 Hurwitz JJ, Maisey MN, Welham RA. Quantitative lacrimal scintillography. I. Method and physiological application. Br J Ophthalmol 1975; 59(6): 308-312.

11 Chavis RM, Welham RA, Maisey MN. Quantitative lacrimal scintillography. Arch Ophthalmol 1978; 96(11): 2066-2068.

12 Carlton WH, Trueblood JH, Rossomondo RM. Clinical evaluation of microscintigraphy of the lacrimal drainage apparatus. J Nucl Med 1973; 14(2): 89-92. 
13 Rossomondo RM, Carlton WH, Trueblood JH, Thomas RP. A new method of evaluating lacrimal drainage. Arch Ophthalmol 1972; 88(5): 523-525.

14 Amanat LA, Hilditch TE, Kwok CS. Lacrimal scintigraphy. II. Its role in the diagnosis of epiphora. Br J Ophthalmol 1983; 67(11): 720-728.

15 Jabbour J, Van der Wall H, Katelaris L, Leslie J, Mackey D, Ghabrial R. Quantitative lacrimal scintigraphy in the assessment of epiphora. Clin Nucl Med 2008; 33(8): 535-541.

16 Fard-Esfahani A, Gholamrezanezhad A, Mirpour S, Tari AS, Saghari M, Beiki D et al. Assessment of the accuracy of lacrimal scintigraphy based on a prospective analysis of patients' symptomatology. Orbit 2008; 27(4): 237-241.

17 Jager PL, Mansour K, Vrakkink-de Zoete H, Poot L, Hooijmans JM, Bruin $\mathrm{KJ}$ et al. Clinical value of dacryoscintigraphy using a simplified analysis. Graefes Arch Clin Exp Ophthalmol 2005; 243(11): 1134-1140.

18 Weidenbecher M, Hosemann W, Buhr W. Endoscopic endonasal dacryocystorhinostomy: results in 56 patients. Ann Otol Rhinol Laryngol 1994; 103(5 Pt 1): 363-367.
19 Kashkouli MB, Parvaresh M, Modarreszadeh M, Hashemi M, Beigi B. Factors affecting the success of external dacryocystorhinostomy. Orbit 2003; 22(4): 247-255.

20 Fayers T, Dolman PJ. Bicanalicular silicone stents in endonasal dacryocystorhinostomy: results of a randomized clinical trial. Ophthalmology 2016; 123(10): 2255-2259.

21 Gencoglu EA, Dursun D, Akova YA, Cengiz F, Yalcin H, Koyuncu A. Tear clearance measurement in patients with dry eye syndrome using quantitative lacrimal scintigraphy. Ann Nucl Med 2005; 19(7): 581-587.

22 Paulsen F, Thale A, Mentlein R. What happens to tears inside the efferent lacrimal passage? An animal experimental study. Graefes Arch Clin Exp Ophthalmol 2000; 238(6): 496-499.

23 Zilelioglu G, Kucuk O, Tekeli O, Gunan F, Aras G. Quantitative lacrimal scintigraphy after dacryocystorhinostomy. Ophthalmic Surg Lasers Imaging 2004; 35(1): 37-40.

24 Mansour K, Blanksma LJ, Vrakking H, Jager PL. Scintigraphic evaluation for tear drainage, after dacryocystorhinostomy, in relation to patient satisfaction. Eye 2008; 22(3): 414-419. 